\title{
Concentration of the Cross-Linked Carboxyterminal Telopeptide of Type I Collagen in Serum of Young Growing Rats Fed a Low Calcium and Vitamin D-Deficient Diet
}

\author{
Adam Robert Bielaczyc ${ }^{1}$, Maria Golebiewska ${ }^{2}$, Anna Citko ${ }^{3}$ and Franciszek Rogowski ${ }^{3}$ \\ 1 Department of Orthodontics \\ 2 Department of Prosthetic Dentistry \\ 3 Department of Nuclear Medicine \\ Medical University, Białystok, Poland
}

\begin{abstract}
Summary: Type I collagen is the main type found in mineralized bone. Specific radioimmunoassay for the crosslinked carboxyterminal telopeptide of type I collagen allows assessing the degradation of type I collagen in serum samples.
\end{abstract}

The aim of the present investigation was to determine the concentration of cross-linked carboxyterminal telopeptide of type I collagen in serum of dietary calcium and vitamin D-deficient rats, a good model disease of decreased formation and mineralization of bone matrix and excessive bone resorption. The studies were carried out on 20 young growing Wistar rats. Serum concentration of the cross-linked carboxyterminal telopeptide of type I collagen was analyzed by the Rat Telopeptide [ ${ }^{125}$ I]ICTP Radioimmunoassay Kit obtained from Orion Diagnostica (Finland).

The data obtained from biochemical analysis showed increased concentration of the cross-linked carboxyterminal telopeptide of type I collagen in the serum of rats fed a low calcium and vitamin D-deficient diet after 14 days of the experiment. At the end of the experiment (day 21), the concentration of carboxyterminal telopeptide of type I collagen in serum was still elevated in these animals.

In conclusion, dietary calcium and vitamin D-deficiency in rats produces hypocalcaemia together with the increased concentration of the cross-linked carboxyterminal telopeptide of type I collagen in serum.

\section{Introduction}

Type I collagen is the most abundant form of collagen in bone, forming $90 \%$ of the organic matrix. It is synthesized by osteoblasts and is formed as a large precursor protein, type I procollagen $(1,2)$. In type I collagen of bones part of the mature cross-links is formed from two original hydroxylysine residues and one lysine residue (deoxypyridinoline) or from three hydroxylysine residues (pyridinoline). The hydroxylysine residues can be glycosylated. Similar structures occur in other collagen types, particularly in the type II collagen of cartilage, which is rich in pyridinoline (3). When the collagen matrix is degraded, both kinds of cross-links are released into the circulation and excreted in urine. Deoxypyridinoline is found almost exclusively in bone, whereas pyridinoline is located in collagen fibrils of bone as well as cartilage. The measurements of deoxypyridinoline and pyridinoline in urine are considered to be indices of the activity of bone collagen degradation $(4,5)$.

Another simple and quantitative test of type I collagen degradation is based on the analysis of the concentration of carboxyterminal telopeptide region of type I

collagen. The telopeptide is liberated during degradation of collagen fibrils of bone and can be estimated in blood samples $(2,3)$. Many diseases, e. g. rheumatoid arthritis, multiple myeloma, bone metastases in patients suffering from cancer of the prostate, and osteopenia among premature infants are associated with increased degradation of type I collagen in the skeleton $(2,6-8)$. Furthermore, long-term suppressive thyroxine treatment in patients with thyroid carcinoma seems to accelerate bone collagen degradation and carries a risk of osteoporosis (9). Type I collagen telopeptide concentration was also found to be correlated with bone resorption rate, measured histomorphometrically $(3,8,10)$.

In the experimental study performed on the rats fed a low calcium and vitamin D-deficient diet for 21 days, the increased resorptive processes concomitant with a defect in the process of bone formation and its mineralization of the alveolar process in histologic examinations were observed. Densitometric analysis showed the decreased density of the whole bony system of these ani- 
mals and finally biochemical examinations showed hypocalcaemia together with the increase in alkaline phosphatase activity (11).

The aim of the present investigation was to determine serum concentration of the cross-linked carboxyterminal telopeptide of type I collagen in rats fed a low calcium and vitamin D-deficient diet.

\section{Materials and Methods}

Animals and diets

Young male Wistar rats (body weight $106 \pm 3.6 \mathrm{~g}, 30$ days old) were the experimental animals. At the beginning of the experimental period, the rats were divided into two groups with 10 animals in each group:

(1) control animals - group B - were fed a diet meeting the nutritional needs of the rats (12 g/kg Ca, 2250 I.U. $/ \mathrm{kg}$ vitamin $\left.\mathrm{D}_{3}\right)$;

(2) test animals - group A - were fed a deficient diet $(0.4 \mathrm{~g} / \mathrm{kg}$ $\mathrm{Ca}$, no vitamin $\mathrm{D})$, but otherwise equal to the diet fed the first group.

The animals were allowed free access to water. The diet was based on the original recipe made by Engström et al. (12).

\section{Specimen collection and storage}

Blood samples were drawn from each animal by intravenous puncture of the jugular vein on days 14 and 21 of the experiment. Serum samples were separated and stored at $-30^{\circ} \mathrm{C}$ until they were assayed. At the end of the experiment (day 21), the animals were killed by exsanguination under anaesthesia with sodium barbital ( $40 \mathrm{mg} / \mathrm{kg}$ intraperitoneal).

Determination of serum concentration of the crosslinked carboxyterminal telopeptide of type I collagen (ICTP)

\section{Principles of the radioimmunoassay}

Concentration of cross-linked carboxyterminal telopeptide of type I collagen in serum was determined by radioimmunoassay (Rat-Telopeptide $\left[{ }^{125} I\right]$ ICTP, Orion Diagnostica, Espoo, Finland). The rat carboxyterminal telopeptide of type I collagen assay kit is based on the widely known radioimmunoassay technique. This assay uses the antigen-antibody reaction where the labelled and non-labelled antigens compete for the limited number of high affinity binding sites of the antibodies. The amount of radioactive antigen in the antigen-antibody complex is inversely proportional to the amount of non-labelled antigen in the reaction mixture. After the separation of the free antigen from the antibody-antigen complex, the residual radioactivity is counted and the actual concentration is calculated with the aid of a standard curve based on the known amounts of non-labelled antigen analyzed parallel to the unknown ones (according to Orion Diagnostica Instruction Kit).

Procedure of the assay

Serum samples $(25 \mu \mathrm{l})$ were mixed with $200 \mu \mathrm{l}$ of RAT-ICTP antiserum and $200 \mu \mathrm{l}$ of ${ }^{125}$ I-labelled RAT-ICTP. After a 2 hour incubation at $37^{\circ} \mathrm{C}$ separation reagent was mixed thoroughly by gentle inversion and then added. Tubes were allowed to stand for $30 \mathrm{~min}-$ utes at room temperature and then were centrifuged for 30 minutes. The supernatant was then removed and the sediment containing the precipitated antibody-antigen complex was counted in a gamma counter (according to Orion Diagnostica Instruction Kit).

In our laboratory, the intra-assay precision ranged from $2.8 \%$ to $6.2 \%$; inter-assay precision ranged from $4.1 \%$ to $7.9 \%$; the sensitivity of the assay was $0.5 \mu \mathrm{g} / \mathrm{l}$. The concentration of the cross-linked carboxyterminal telopeptide of type I collagen in serum was expressed in $\mu \mathrm{g} / \mathrm{l}$.

\section{Determination of total calcium concentration in serum}

Total calcium concentration in sera of the rats from both groups were determined by standard colorimetric methods using a Cobas Mira analyzer, Hoffmann LaRoche (Switzerland).

\section{Statistics}

The data obtained from biochemical study were presented as the arithmetical mean with the standard deviation $(x \pm$ SD) for each group of 10 rats $(n=10)$. A comparison between the two groups was performed with the unpaired Student's t test. Comparisons between the mean values of serum concentration of the cross-linked carboxyterminal telopeptide of type I collagen obtained on days 14 and 21 of the experiment within one group were performed with the paired Student's test. Statistically significant difference was considered at $\mathrm{p}<0.05$.

\section{Results}

The results of biochemical analysis of total calcium concentration in sera of the rats fed a low calcium and vitamin D-deficient diet had been presented previously (6). The mean value of total calcium concentration in sera of the test animals obtained on day 14 was $1.84 \pm 0.05 \mathrm{mmol} / \mathrm{l}$, while in the control animals it was $2.51 \pm 0.12 \mathrm{mmol} / \mathrm{l}$. The differences between the values were statistically significant $(p<0.001)$. On day 21 , the mean value of total calcium concentration in sera of the test animals was $1.78 \pm 0.1 \mathrm{mmol} / \mathrm{l}$ and was lower than that obtained in the control rats $-2.53 \pm 0.11 \mathrm{mmol} /$ 1. The differences between the values were statistically significant $(\mathrm{p}<0.001)$.

The concentration of the cross-linked carboxyterminal telopeptide of type I collagen on days 14 and 21 of the experiment are shown in table 1 . The mean concentration of the cross-linked carboxyterminal telopeptide of type I collagen in serum on day 14 was significantly higher in the rats fed a low calcium and vitamin Ddeficient diet $(p<0.001)$ in comparison to the mean value obtained in the control animals. At the end of the experiment (day 21), the concentration of the carboxyterminal telopeptide of type I collagen in serum was still

Tab. 1 Mean values of serum carboxyterminal telopeptide of type I collagen concentration in the test (group A) and the control (group B) animals (days 14 and 21). Values are the mean with the standard deviation $(x \pm S D)$ for the groups of 10 animals each $(\mathrm{n}=10)$

\begin{tabular}{lll}
\hline & \multicolumn{2}{l}{$\begin{array}{l}\text { Concentration of carboxyterminal } \\
\text { telopeptide of type I collagen }[\mu \mathrm{g} / \mathrm{l}]\end{array}$} \\
\cline { 2 - 3 } & Day 14 & Day 21 \\
\hline $\begin{array}{l}\text { Group A } \\
\mathrm{n}=10\end{array}$ & $\begin{array}{l}24.08 \pm 1.9^{*} \\
\mathrm{p}<0.001\end{array}$ & $\begin{array}{l}\mathrm{p}<0.19 \pm 2.5 \\
\text { Group B } \\
\mathrm{n}=10\end{array}$ \\
& $19.03 \pm 1.0$ & $19.11 \pm 2.43$
\end{tabular}

* $p<0.001$ statistically significant difference between the mean values obtained on days 14 and 21 of the study in test animals (group A) 
elevated in the test animals $(p<0.001)$ compared to the mean value obtained in the control group. A statistically significant difference $(p<0.001)$ was also found between the mean values of serum concentration of the cross-linked carboxyterminal telopeptide of type I collagen in the test animals obtained on days 14 and 21 of the study.

\section{Discussion}

Bone remodelling first comprises a phase of osteoclast activation and osteoclastic bone resorption with the formation of a resorption cavity. Subsequently, osteoblasts synthesize type I collagen. Then the osteoid matrix thus formed undergoes mineralization and self-repair of skeletal tissue occurs (2). A dietary calcium and vitamin Ddeficiency in rats enhances osteoclastic bone resorption concomitant with a defect in bone matrix formation and its mineralization as observed in our previous histologic examinations. We observed these morphological changes in the alveolar process of the rat's maxilla since this tissue is characterized by the high bone turnover (11).

Type I collagen telopeptide concentration was found to be correlated with cancellous bone resorption rate, measured by histomorphometry, and therefore is regarded as a specific marker of bone resorption $(8,10)$.

Elomaa et al. (2) studied the serum concentration of carboxyterminal propeptide of type I procollagen and carboxyterminal telopeptide of type I collagen in order to follow bone turnover and evaluate treatment response in multiple myeloma, which is a good model disease for excessive osteolysis. The study indicated that serum concentration of carboxyterminal telopeptide of type I collagen correlated with the number of osteolytic lesions. The lack of correlation between carboxyterminal telopeptide of type I collagen and carboxyterminal propeptide of type I procollagen proves, in the authors' opinion, imbalanced bone turnover and suggests unchanged and even depressed bone formation in multiple myeloma.

Eriksen at al. (10), using the method of calcium kinetics and dynamic histomorphometry, found a significant correlation between serum concentration of carboxyterminal telopeptide of type I collagen and bone resorption in patients with low and high bone turnover rates.

Mora et al. (8) stated that carboxyterminal telopeptide of type I collagen is a useful marker of bone modelling in osteopenia, a well-recognized disorder of bone metabolism among premature infants. The authors studied bone formation and degradation through the measurements of three indices: osteocalcin, carboxyterminal propeptide of type I procollagen and carboxyterminal telopeptide of type I collagen in premature infants at the age of 4 weeks in comparison to full-term subjects. The results showed significantly higher osteocalcin levels in full-term infants, while the bone resorption marker, carboxyterminal telopeptide of type I collagen, was significantly higher in premature infants than in full-term ones. Carboxyterminal propeptide of type I procollagen did not differ between the two groups. The authors conclude that in premature infants at the age of 4 weeks, there appeared a low osteoblastic activity and enhanced bone degradation compared with that of full-term controls. The imbalance of bone modelling could be the cause of the reduced bone mineralization found in preterm infants.

Hakala et al. (6) assessed the extent and clinical significance of serum carboxyterminal telopeptide of type I collagen in rheumatoid arthritis. It has been proven by the authors that the elevated serum concentration of carboxyterminal telopeptide of type I collagen is common in rheumatoid arthritis and associated with the signs of aggressive disease.

To the contrary, Garnero et al. (13) performed studies on a group consisting of late postmenopausal osteoporotic women and found no statistically significant differences in the values of serum concentration of carboxyterminal telopeptide of type I collagen and carboxyterminal propeptide of type I procollagen in comparison with the group of controls, although other biochemical markers of bone metabolism, e. g. serum total and serum intact osteocalcin concentration, serum bone alkaline phosphatase activity and urinary total pyridinoline and deoxypyridinoline, indicated increased bone turnover and, as determined by dual energy X-ray absorptiometry showed decreased bone mass. The authors explain that a possible reason for inability of serum carboxyterminal telopeptide of type I collagen to detect increase in bone turnover is that the circulating carboxyterminal telopeptide of type I collagen may not reflect the true collagen degradation rate, but may reflect other aspects of connective tissue turnover. Delmas (14) suggests that serum carboxyterminal telopeptide of type I collagen is an index of collagen turnover, but not a specific marker of bone resorption.

The results of our study showed the increased values of serum concentration of the cross-linked carboxyterminal telopeptide of type I collagen in the rats fed a low calcium and vitamin D-deficient diet in comparison to the values obtained in the control animals. Considering the morphological changes of the alveolar process of the maxilla and densitometric data described previously (11) we suggest that the elevated serum concentration of the cross-linked carboxyterminal telopeptide of type I collagen correlates with the increased bone resorption and decreased density of the whole bony system in the rats fed a low calcium and vitamin D-deficient diet. More- 
over, the elevated serum concentration of the crosslinked carboxyterminal telopeptide of type I collagen correlates with the decreased levels of total calcium in sera of these animals.

\section{References}

1. Cinaz P, Hasanoglu E, Gökcora N, Dogukan S, Demir A. Serum levels of carboxyterminal propeptide of type I procollagen and aminoterminal propeptide of type III procollagen in children with growth retardation. Mater Med Pol 1994; 26:55 -8.

2. Elomaa I, Virkkunen L, Risteli J. Serum concentration of the cross-linked carboxyterminal telopeptide of type I collagen (ICTP) is a useful prognostic indicator in multiple myeloma. Br J Cancer 1992; 66:337-41.

3. Risteli J, Elomaa I, Niemi S, Novamo A, Risteli L. Radioimmunoassay for the pyridinoline cross-linked carboxyterminal telopeptide of type I collagen: a new serum marker of bone collagen degradation. Clin Chem 1993; 39:635-40.

4. Withold W, Georgescu G, Khakzad H, Vosberg H, MuellerGaertner HW, Reinauer H. Simultaneous assessment of bone collagen synthesis and degradation in patients with different malignant tumours in comparison with the results of ${ }^{99 \mathrm{~m}} \mathrm{Tc}$ methylene bisphosphonate bone scintigraphy. Eur J Clin Chem Clin Biochem 1995; 33:479-85.

5. Withold W, Degenhardt S, Heins M, Grabensee B, Reinauer $\mathrm{H}$. Monitoring of bone resorption after renal transplantation by measuring the urinary excretion of pyridinium cross-links. Eur J Clin Chem Clin Biochem 1995; 33:15-21.

6. Hakala M, Risteli L, Manelius J, Nieminen P, Risteli J. Increased type I collagen degradation correlates with disease severity in rheumatoid arthritis. Ann Rheum Dis 1993; 52:866-9.

7. Westerhuis LWJJM, Delaere KPJ. Diagnostic value of some biochemical bone markers for the detection of bone metastases in prostate cancer. Eur J Clin Chem Clin Biochem 1997; 35(2):89-94.
In conclusion, dietary calcium and vitamin D-deficiency in rats produces hypocalcaemia together with the increased serum concentration of the cross-linked carboxyterminal telopeptide of type I collagen.

8. Mora S. Bone modeling alternation in premature infants. Arch Pediatr Adolesc Med 1994; 148:1215-7.

9. Taimela E, Taimela S, Nikkanen V, Irjala K. Accelerated bone degradation in thyroid carcinoma patients during thyroxine treatment, measured by determination of the carboxyterminal telopeptide region of type I collagen in serum. Eur J Clin Chem Clin Biochem 1994; 32:827-31.

10. Eriksen EF, Melsen CP, Mosekilde L, Risteli L, Risteli J. Serum markers of type I collagen formation and degeneration in metabolic bone disease: correlation to bone histomorphometry. J Bone Miner Res 1993; 8:127-32.

11. Bielaczyc A. The histological picture of rat periodontium with systemic calcium deficiency. Czas Stomatol 1997; L, 2:110-8.

12. Engström C, Linde A, Magnusson BC. Odontoblast metabolism in rats deficient in vitamin $\mathrm{D}$ and calcium $\mathrm{I}$ : a histochemical survey. J Oral Pathol 1977; 6:359-66.

13. Garnero P, Shih W J, Gineyts E, Karpf DB, Delmas PD. Comparison of new biochemical markers of bone turnover in late postmenopausal osteoporotic women in response to alendronate treatment. J Clin Endocrinol Metab 1994; 79:1693-700.

14. Delmas PD. Biochemical markers for the assessment of bone turnover. In: Riggs BL, Melton LJ, editors. Osteoporosis: etiology and diagnosis and management. Philadelphia: LippincottRaven Publ. 1995:319-33.

\section{Received July 11/ September 30, 1997}

Corresponding author: Dr n. med. Maria Gołębiewska, Department of Prosthetic Dentistry, Medical University, Białystok, M. C. Skłodowskiej 24a, PL-15-276 Białystok, Poland 